\title{
Chromone-3-carboxaldehydes in Passerini Reactions Using TosMIC as the Isonitrile Component
}

\author{
Michael A. Terzidis, Julia Stephanidou-Stephanatou and Constantinos A. Tsoleridis*
}

Department of Chemistry, Laboratory of Organic Chemistry, University of Thessaloniki, Thessaloniki 54124, Greece

\begin{abstract}
Toluenesulfonylmethyl isocyanide (TosMIC) is used as the isonitrile component in Passerini reactions with chromone-3-carboxaldehydes to yield chromenyl-amidoesters in good yields.
\end{abstract}

Keywords: Chromenyl-acetamides, chromone-3-carboxaldehydes, Passerini reaction, three component reaction, TosMIC

Besides forming the basic nucleus of an entire class of natural products, i.e. flavones [1], the chromone moiety forms the important component of pharmacophores of a large number of molecules of medicinal significance [2] including anticancer agents, such as psorospermin and pluramycin A $[3,4]$. Consequently, considerable attention is being devoted to isolation from natural resources, chemistry and synthesis of chromone derivatives, and evaluation of their biological activity with stress on their potential medicinal applications [2-6]. Chromone-3-carboxaldehyde represents a very reactive system owing to the presence of an unsaturated keto function, a conjugated second carbonyl group at C-3 and of a reactive center at C-2. Therefore, chromone- isocyanides are very versatile reactions [12] in terms of scaffolds and number of accessible compounds. Amongst these reactions, the Passerini [13] multicomponent reaction occupies an important position. However, the synthetic utility of TosMIC as an isonitrile input in a Passerini reaction remains yet underexploited, although further advantages could derive from the fact that the products, besides being diversely functionalized, also bear an additional methylene group, which can serve as a handle for further manipulation. Only very recently, Krisna et al. [14] studied the use of TosMIC as the isonitrile component in Passerini reactions with 2,3-epoxyand sugar derived aldehydes. TosMIC has most commonly been used [15] in heterocyclic ring construction, in particular<smiles>[R]c1cc2occ(C=O)c(=O)c2cc1[R]</smiles><smiles>[R]C(=O)OC(C(=O)NCCCCCCCCCC)c1coc2cc([R])c([R])cc2c1=O</smiles>

\begin{tabular}{lll} 
& $\mathrm{R}^{1}$ & \multicolumn{2}{c}{$\mathrm{R}^{2}$} \\
\cline { 2 - 3 } a & $\mathrm{H}$ & $\mathrm{H}$ \\
b & $\mathrm{Me}$ & $\mathrm{H}$ \\
c & $\mathrm{Cl}$ & $\mathrm{H}$ \\
d & $\mathrm{Cl}$ & $\mathrm{Me}$ \\
e & $\mathrm{NO}_{2}$ & $\mathrm{H}$
\end{tabular}

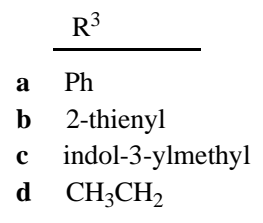

\begin{tabular}{llll} 
& $\mathrm{R}^{1}$ & $\mathrm{R}^{2}$ & $\mathrm{R}^{3}$ \\
\cline { 2 - 5 } a & $\mathrm{H}$ & $\mathrm{H}$ & $\mathrm{Ph}$ \\
b & $\mathrm{Me}$ & $\mathrm{H}$ & $\mathrm{Ph}$ \\
c & $\mathrm{Cl}$ & $\mathrm{H}$ & $\mathrm{Ph}$ \\
d & $\mathrm{Cl}$ & $\mathrm{Me}$ & $\mathrm{Ph}$ \\
e & $\mathrm{NO}_{2}$ & $\mathrm{H}$ & $\mathrm{Ph}$
\end{tabular}

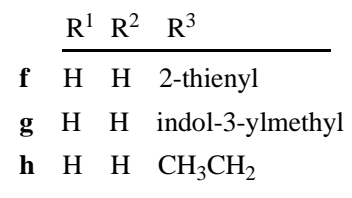

Scheme 1. Reaction of chromone-3-carboxaldehydes 1 with TosMIC in the presence of carboxylic acids 2.

3-carboxaldehydes can be readily converted into a broad range of heterocyclic systems either by cycloaddition strategies $[7,8]$ or through reaction with several nucleophiles $[9,10]$.

Moreover, the rich and fascinating chemistry that stems from multicomponent reactions (MCRs) provides [11] a powerful tool towards the one-pot synthesis of diverse and complex compounds on the one hand and small and 'druglike' heterocycles on the other hand. MCRs that involve

*Address correspondence to this author at the Department of Chemistry, Laboratory of Organic Chemistry, University of Thessaloniki, Thessaloniki 54124, Greece; Tel: +30-2310-997865; Fax: +30-2310-997679;

E-mail: tsolerid@chem.auth.gr of oxazole and pyrrole moieties. Recently, we published the 2-tosyl-4-(2-hydroxybenzoyl) pyrrole synthesis mediated by the reaction between chromone-3-carboxaldehydes with TosMIC [16]. This work motivated us to extrapolate the synthetic utility of TosMIC as an isonitrile moiety in three component reactions involving chromone-3-carboxaldehydes, aiming at the isolation of chromenyl-amidoesters, in view of the diverse biological activity shown by 3-substituted chromone derivatives [17].

When a methylene chloride solution of the chromone-3carboxaldehydes $(\mathbf{1 a}-\mathbf{1 e})$ was allowed to react with equimolar amounts of benzoic acid and TosMIC 2-benzoyloxy-2-(4oxo-4H-chromen-3-yl)- $N$ - $p$-tosylmethyl-acetamides $(\mathbf{3 a}-\mathbf{3 e})$ were isolated in very good yields $70-85 \%$ (Scheme 1, Table 
Table 1. Reactants and Products

\begin{tabular}{|c|c|c|c|c|}
\hline Entry & Chromone & Acid & Prod & 3a \\
\hline \hline 1 & $\mathbf{1 a}$ & $\mathbf{2 a}$ & $\mathbf{3 b}$ & $\mathbf{2 a}$ \\
\hline 2 & $\mathbf{1 b}$ & $\mathbf{2 a}$ & $\mathbf{3 c}$ & $\mathbf{3 0}$ \\
\hline 3 & $\mathbf{1 c}$ & $\mathbf{2 a}$ & $\mathbf{3 e}$ & $\mathbf{3 0}$ \\
\hline 4 & $\mathbf{1 d}$ & $\mathbf{2 a}$ & $\mathbf{3 f}$ & 30 \\
\hline 5 & $\mathbf{1 e}$ & $\mathbf{2 b}$ & $\mathbf{3 g}$ & 32 \\
\hline 6 & $\mathbf{1 a}$ & $\mathbf{2 c}$ & $\mathbf{3 h}$ & 29 \\
\hline
\end{tabular}

1) $[18,19]$. The reaction proceeded successfully also with acids containing a heterocyclic ring, such as 2-thiophenyl and 3-indolylacetic acids, though in lower yields. Having established a positive outcome of the Passerini reaction by using an aromatic acid, in order to examine the generality of the reaction, the reaction was repeated with the aliphatic propionic acid. The reaction proceeded uniformly to afford product 3h, but in comparatively low yield (29\%, Table 1).

Next, we also studied some possible transformations of the isolated chromenyl-amido-esters $\mathbf{3}$. For this reason $\mathbf{3 a}$ and $\mathbf{3 c}$ were stirred at room temperature in ethanolic sodium hydroxide solution for $24 \mathrm{~h}$, whereupon through a double nucleophilic substitution the chromenyl-acetamides $\mathbf{4 a}$ and 4c were isolated in $71 \%$ and $74 \%$ yield, respectively (Scheme 2) [20].

The assigned molecular structures of all new compounds 3 are based on rigorous spectroscopic analysis including IR, NMR $\left({ }^{1} \mathrm{H},{ }^{13} \mathrm{C}\right.$, COSY, NOESY, HETCOR and COLOC), MS and elemental analysis data. Regarding the structure of products $\mathbf{3}$ the assignment of $\mathbf{3 b}$ is described. The elemental analysis and mass spectra unequivocally established the reaction of one molecule of chromone-carboxaldehyde $\mathbf{1 b}$ with one molecule of TosMIC and one molecule of benzoic acid, a fact that was also confirmed from the ${ }^{13} \mathrm{C}$ NMR spectrum, where 23 different signals were observed. Moreover, in the IR spectrum besides the carbonyl absorptions at 1702, 1682 and $1655 \mathrm{~cm}^{-1}$ an $\mathrm{NH}$ absorption appeared at $3379 \mathrm{~cm}^{-1}$. From the H-H COSY spectrum three distinguishable proton groups were defined corresponding to the three aromatic rings. In the ${ }^{1} \mathrm{H}$ NMR the presence of the tosyl group was identified from the three proton singlet at $\delta 2.30$ (with the corresponding carbon at $21.6 \mathrm{ppm}$ ) and the $p$-substituted phenyl moiety $\left(\delta \mathrm{H}_{2^{\prime \prime}, 6^{\prime \prime \prime}}=7.60, \delta \mathrm{H}_{3^{\prime \prime}, 5^{\prime \prime \prime}}=6.99\right.$, with corresponding carbons resonating at 128.9 and $129.6 \mathrm{ppm}$, respec- tively) [19]. The Tos-methyl group protons gave COLOC correlations with the carbon at $129.6 \mathrm{ppm}$ and also with the quaternary carbon at $145.0 \mathrm{ppm}$, whereas the protons at $\delta$ 6.99 correlated with the quaternary carbon at $133.7 \mathrm{ppm}$. The TosMIC methylene protons, due to restricted rotation of the tosyl group, appeared as two doublet of doublets at $\delta 4.50(\mathrm{~J}$ $=14.25,5.25 \mathrm{~Hz})$ and $\delta 5.00(J=14.25,8.45 \mathrm{~Hz})$ with corresponding carbon at $60.0 \mathrm{ppm}$, due to additional coupling with the neighboring $\mathrm{NH}$ group $(\delta$ 7.86). The methylene protons correlated with a carbonyl carbon at $167.4 \mathrm{ppm}$, being the former isonitrile carbon, so the whole sequence of the TosMIC reactant was identified, as depicted in Fig. (1). Concerning the chromone aromatic moiety, this was identified from the splitting pattern of the aromatic protons, resonating as a doublet at $\delta 8.06(J=1.3 \mathrm{~Hz})$, a multiplet at $\delta 7.56-$ 7.63 , and a doublet at $\delta 7.44(J=8.4 \mathrm{~Hz})$ and their carbons resonating at $125.3,136.0$ and $118.0 \mathrm{ppm}$, respectively. The chromone methyl group proton singlet appeared at $\delta 2.51$ (with carbon at $21.1 \mathrm{ppm}$ ), whereas the 2 '-position proton appeared as a doublet at $\delta 8.10$, coupled with the C-2 aliphatic proton by allylic coupling $(J=0.75 \mathrm{~Hz})$ with the corresponding carbon resonating at $155.0 \mathrm{ppm})$. This proton showed COLOC correlations with the chromone carbonyl carbon at $176.4 \mathrm{ppm}$ and also with the quaternary carbons at $118.7\left(\mathrm{C}-3^{\prime}\right)$ and $154.6\left(\mathrm{C}-8 \mathrm{a}^{\prime}\right)$ via ${ }^{2} J_{\mathrm{C}-\mathrm{H}}$ and ${ }^{3} J_{\mathrm{C}-\mathrm{H}}$. couplings. With the last carbon at $154.6 \mathrm{ppm}$ is correlated also the $7^{\prime}$ position proton. Moreover, the one proton signal which appeared as a doublet at $\delta 6.29(J=0.75 \mathrm{~Hz}, \mathrm{C} 68.4 \mathrm{ppm})$ correlated with the three carbonyl carbons at 165.0, 167.4 and 176.4 and also with the $\mathrm{C}-2^{\prime}$ protonated carbon, indicating thus its correspondence to the former formyl proton. Finally, the five phenyl ring protons showed the expected characteristic proton and carbon absorptions, in addition to the carbonyl carbon at $165.0 \mathrm{ppm}$.

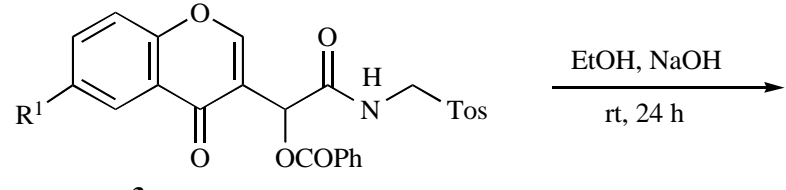
3

a $\quad \mathrm{R}^{1}=\mathrm{H} ;$ c $\mathrm{R}^{1}=\mathrm{Cl}$<smiles>[R]COCNC(=O)C(OCC)c1coc2ccc([R])cc2c1=O</smiles>

4

Scheme 2. Reaction of chromonyl amidoester 3a and 3c with ethanolic $\mathrm{NaOH}$. 


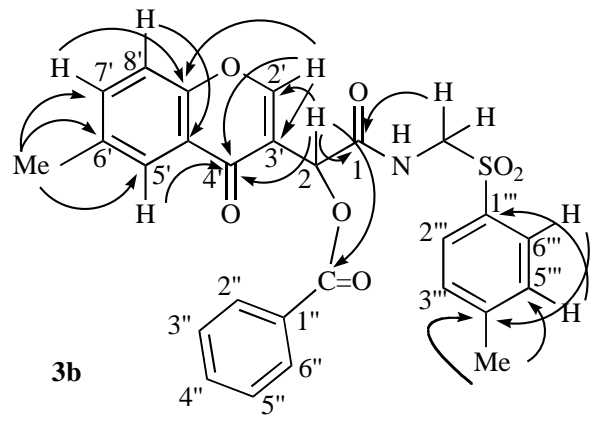

Fig. (1). Diagnostic COLOC correlations between protons and carbons (via ${ }^{2} J_{\mathrm{C}-\mathrm{H}}$ and ${ }^{3} J_{\mathrm{C}-\mathrm{H}}$ ), and position numbering in compound $\mathbf{3 b}$.

In some spectra a characteristic allylic coupling between the $\mathrm{C}(2)-\mathrm{H}$ and $\mathrm{C}\left(2^{\prime}\right)-\mathrm{H}$ of $\sim 0.6-0.8 \mathrm{~Hz}$ is observed. Moreover, in all compounds the $\mathrm{NHCH}_{2}$ methylene protons show $\mathrm{AB}$ patern with geminal coupling of $\sim 14 \mathrm{~Hz}$ in addition to two distinctly defined coupling constants of $\sim 8 \mathrm{~Hz}$ and $\sim 5$ $\mathrm{Hz}$, due to simultaneous coupling with the NH proton, confirming thus the configuration in molecular model depicted in Fig. (2) (after PM3 calculation).

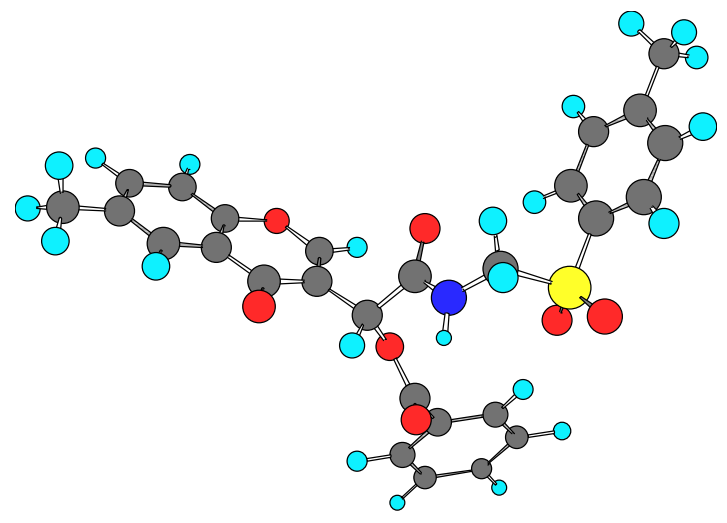

Fig. (2). Molecular model calculated for compound 3b (PM3).

In conclusion, we have studied a Passerini reaction involving chromones and TosMIC leading to the isolation of unknown chromenyl-amidoesters 3 . The transformation of the isolated chromenyl-amidoesters $\mathbf{3}$ to chromenylacetamides 4 in ethanolic sodium hydroxide solution was also examined. Work is continuing in our laboratory in this area and we will report on further studies in the future.

\section{REFERENCES}

[1] (a) Dewick, P. M. In The Flavonoids: Advances in Research Since 1986; Harborne, J. B., Ed.; Chapman \& Hall: New York, NY, 1994, pp. 117-238. (b) Gill, M. In The Chemistry of Natural Products, 2nd ed.; Thomson, R. H., Ed.; Blackie: Surrey, 1993, pp. 60105. (c) Flavonoids in the Living Systems: Advances in Experimental Medicine and Biology; Manthey, J. A.; Buslig, B. S., Eds.; Plenum: New York, 1998, Vol. 439.

[2] (a) Korkina, G. L.; Afanas'ev, I. B. Advances in Pharmacology; Sies, H., Ed.; Academic Press: San Diego, CA, 1997, Vol. 38, pp. 151-163. (b) Comprehensive Medicinal Chemistry; Hansch, C.; Sammes, P. G.; Taylor, J. B., Eds.; Pergamon: New York, NY, 1990, Vol. 6.

[3] (a) Kim, M. Y.; Na, Y.; Vankayalapati, H.; Gleason-Guzman, M.; Hurley, L. H. J. Med. Chem., 2003, 46, 2958. (b) Mitscher, L. A. Chem. Rev., 2005, 105, 559.

[4] (a) Cassady, J. M.; Baird, W. M.; Chang, C. J. J. Nat. Prod., 1990, 53, 23. (b) Kupchan, S. M.; Streelman, D. R.; Sneden, A. T. J. Nat. Prod., 1980, 43, 296.

(1)
$7.02\left(\mathrm{~d}, J=8.0 \mathrm{~Hz}, 2 \mathrm{H}, \mathrm{H}-3^{\prime \prime \prime}, 5^{\prime \prime \prime}\right), 7.45-7.50\left(\mathrm{~m}, 2 \mathrm{H}, \mathrm{H}-3^{\prime \prime}, 5^{\prime \prime}\right)$,
$7.53\left(\mathrm{ddd}, J=8.0,7.0,1.0 \mathrm{~Hz}, 1 \mathrm{H}, \mathrm{H}-6^{\prime}\right), 7.55(\mathrm{~d}, J=8.5 \mathrm{~Hz}, 1 \mathrm{H}$, $\left.\mathrm{H}-8^{\prime}\right), 7.62\left(\mathrm{tt}, J=7.5,1.25 \mathrm{~Hz}, 1 \mathrm{H}, \mathrm{H}-4^{\prime \prime}\right), 7.63(\mathrm{~d}, J=8.0 \mathrm{~Hz}, 2 \mathrm{H}$, $\left.\mathrm{H}-2^{\prime \prime \prime}, 6^{\prime \prime \prime}\right), 7.75$ (br m, 1H, NH), 7.79 (ddd, $J=8.5,7.0,1.75 \mathrm{~Hz}$, $\left.1 \mathrm{H}, \mathrm{H}-7^{\prime}\right), 8.05-8.08$ (m, 2H, H-2",6"), 8.14 (d, $J=0.75 \mathrm{~Hz}, 1 \mathrm{H}$, $\left.\mathrm{H}-2^{\prime}\right), 8.29\left(\mathrm{dd}, J=8.0,1.7 \mathrm{~Hz}, 1 \mathrm{H}, \mathrm{H}-5^{\prime}\right) .{ }^{13} \mathrm{C} \mathrm{NMR:}\left(\mathrm{CDCl}_{3}, 75\right.$ $\mathrm{MHz}) 21.6\left(4^{\prime \prime \prime}-\mathrm{CH}_{3}\right), 60.0\left(\mathrm{~N}-\mathrm{CH}_{2}\right), 68.5(\mathrm{C}-2), 118.3\left(\mathrm{C}-8^{\prime}\right)$, $118.9\left(\mathrm{C}-3^{\prime}\right), 123.8\left(4 \mathrm{a}^{\prime}\right), 126.06\left(5^{\prime}\right), 126.13\left(6^{\prime}\right), 128.7\left(\mathrm{C}-3^{\prime \prime}, 5^{\prime \prime}\right)$, 128.9 (C-2"',6"'), $129.7\left(\mathrm{C}-3^{\prime \prime \prime}, 5^{\prime \prime \prime}\right), 130.0\left(\mathrm{C}-2^{\prime \prime}, 6^{\prime \prime}\right), 133.5\left(\mathrm{C}-1^{\prime \prime}\right)$, $133.7\left(1^{\prime \prime \prime}\right), 133.9\left(\mathrm{C}-4^{\prime \prime}\right), 134.7\left(\mathrm{C}-7^{\prime}\right), 145.1\left(\mathrm{C}-4^{\prime \prime \prime}\right), 155.2\left(\mathrm{C}-2^{\prime}\right)$, 156.3 (C-8a'), 165.0 (2-CO), 167.3 (C-1), 176.4 (C-4'). MS (LCMS) m/z (\%) $514\left(100, \mathrm{M}^{+*}+23\right)$. Anal. Calcd for $\mathrm{C}_{26} \mathrm{H}_{21} \mathrm{NO}_{7} \mathrm{~S}$ (491.51): C, 63.53; H, 4.31; N, 2.85\%. Found: C, 63.70; H, 4.17, N, $2.71 \%$.

Data of Compound 3b. Yield $0.368 \mathrm{~g}, 70 \%$, white solid, mp 194$196{ }^{\circ} \mathrm{C}$. IR (nujol) $v_{\max }: 3379,1702,1682,1655 \mathrm{~cm}^{-1} .{ }^{1} \mathrm{H}$ NMR: $\left(\mathrm{CDCl}_{3}, 300 \mathrm{MHz}\right) \delta: 2.30\left(\mathrm{~s}, 3 \mathrm{H}, 4^{\prime \prime \prime \prime}-\mathrm{CH}_{3}\right), 2.51\left(\mathrm{~s}, 3 \mathrm{H}, 6^{\prime}-\mathrm{CH}_{3}\right)$, $4.50\left(\mathrm{dd}, J=14.25,5.25 \mathrm{~Hz}, 1 \mathrm{H}, \mathrm{N}-\mathrm{CH}_{2}\right), 5.00(\mathrm{dd}, J=14.25,8.45$ $\left.\mathrm{Hz}, 1 \mathrm{H}, \mathrm{N}-\mathrm{CH}_{2}\right), 6.29$ (d, $\left.J=0.75 \mathrm{~Hz}, 1 \mathrm{H}, \mathrm{H}-2\right), 6.99$ (d, $J=8.2$ $\left.\mathrm{Hz}, 2 \mathrm{H}, \mathrm{H}-3^{\prime \prime \prime}, 5^{\prime \prime \prime}\right), 7.44$ (d, $\left.J=8.4 \mathrm{~Hz}, 1 \mathrm{H}, \mathrm{H}-8^{\prime}\right), 7.47$ (m, 2H, H$\left.3^{\prime \prime}, 5^{\prime \prime}\right), 7.56-7.63\left(\mathrm{~m}, 2 \mathrm{H}, \mathrm{H}-7^{\prime}, 4^{\prime \prime}\right), 7.60(\mathrm{~d}, J=8.2 \mathrm{~Hz}, 2 \mathrm{H}, \mathrm{H}-$ $\left.2^{\prime \prime \prime}, 6^{\prime \prime \prime}\right), 7.87$ (br dd, $\left.J=8.4,5.3 \mathrm{~Hz}, 1 \mathrm{H}, \mathrm{NH}\right), 8.04-8.07$ (m, 2H, $\left.\mathrm{H}-2^{\prime \prime}, 6^{\prime \prime}\right), 8.06\left(\mathrm{~d}, J=1.3 \mathrm{~Hz}, 1 \mathrm{H}, \mathrm{H}-5^{\prime}\right), 8.10(\mathrm{~d}, J=0.75 \mathrm{~Hz}, 1 \mathrm{H}$, $\left.\mathrm{H}-2^{\prime}\right) .{ }^{13} \mathrm{C}$ NMR: $\left(\mathrm{CDCl}_{3}, 75 \mathrm{MHz}\right) 21.1\left(6^{\prime}-\mathrm{CH}_{3}\right), 21.6\left(4^{\prime \prime \prime}-\mathrm{CH}_{3}\right)$, $60.0\left(\mathrm{~N}-\mathrm{CH}_{2}\right), 68.4(\mathrm{C}-2), 118.0\left(\mathrm{C}-8^{\prime}\right), 118.7\left(\mathrm{C}-3^{\prime}\right), 123.4\left(4 \mathrm{a}^{\prime}\right)$, $125.3\left(5^{\prime}\right), 128.6\left(\mathrm{C}-3^{\prime \prime}, 5^{\prime \prime}\right), 128.9\left(\mathrm{C}-2^{\prime \prime \prime}, 6^{\prime \prime \prime}\right), 129.6\left(\mathrm{C}-3^{\prime \prime \prime}, 5^{\prime \prime \prime}\right)$, $129.9\left(\mathrm{C}-2^{\prime \prime}, 6^{\prime \prime}\right), 133.7\left(1^{\prime \prime \prime}\right), 133.8\left(\mathrm{C}-4^{\prime \prime}\right), 136.0\left(\mathrm{C}-7^{\prime}\right), 136.3(\mathrm{C}-$ $\left.6^{\prime}\right), 136.3\left(\mathrm{C}-1^{\prime \prime}\right), 145.0\left(\mathrm{C}-4^{\prime \prime \prime}\right), 154.6\left(\mathrm{C}-8 \mathrm{a}^{\prime}\right), 155.0\left(\mathrm{C}-2^{\prime}\right), 165.0$ (2-CO), 167.4 (C-1), 176.4 (C-4'). MS (LCMS) m/z (\%) 528 (100, 
$\mathrm{M}^{+*}$ + 23). Anal. Calcd for $\mathrm{C}_{27} \mathrm{H}_{23} \mathrm{NO}_{7} \mathrm{~S}$ (505.54): C, 64.15; H, 4.59; $\mathrm{N}, 2.77 \%$. Found: C, $64.00 ; \mathrm{H}, 4.52, \mathrm{~N}, 2.65 \%$.

Data of Compound 3c. White solid, mp $223-225^{\circ} \mathrm{C}$. IR (nujol) $v_{\max }: 3320,1707,1646 \mathrm{~cm}^{-1} .{ }^{1} \mathrm{H}$ NMR: $\left(\mathrm{CDCl}_{3}, 300 \mathrm{MHz}\right) \delta: 2.33$ (s, 3H, 4"'- $\left.-\mathrm{CH}_{3}\right), 4.55\left(\mathrm{dd}, J=14.2,5.8 \mathrm{~Hz}, 1 \mathrm{H}, \mathrm{N}-\mathrm{CH}_{2}\right), 4.94$ (dd, $\left.J=14.2,8.0 \mathrm{~Hz}, 1 \mathrm{H}, \mathrm{N}-\mathrm{CH}_{2}\right), 6.25(\mathrm{~d}, J=0.5 \mathrm{~Hz}, 1 \mathrm{H}, \mathrm{H}-2), 7.10$ (d, $\left.J=8.0 \mathrm{~Hz}, 2 \mathrm{H}, \mathrm{H}-3^{\prime \prime \prime}, 5^{\prime \prime \prime}\right), 7.43-7.50$ (m, 2H, H-3", 5"), 7.49 (d, $\left.J=9.0 \mathrm{~Hz}, 1 \mathrm{H}, \mathrm{H}-8^{\prime}\right), 7.70$ (dd, $\left.J=9.0,2.5 \mathrm{~Hz}, 1 \mathrm{H}, \mathrm{H}-7^{\prime}\right), 7.62$ (tt, $\left.J=7.3,1.7 \mathrm{~Hz}, 1 \mathrm{H}, \mathrm{H}-4^{\prime \prime}\right), 7.66\left(\mathrm{~d}, J=8.0 \mathrm{~Hz}, 2 \mathrm{H}, \mathrm{H}-2^{\prime \prime \prime}, 6^{\prime \prime \prime}\right)$, 7.60-7.70 (br m 1H, NH), 8.04-8.07 (m, 2H, H-2", $\left.6^{\prime \prime}\right), 8.15$ (d, $J=$ $\left.0.5 \mathrm{~Hz}, 1 \mathrm{H}, \mathrm{H}-2^{\prime \prime}\right), 8.22\left(\mathrm{~d}, J=2.5 \mathrm{~Hz}, 1 \mathrm{H}, \mathrm{H}-5^{\prime}\right) .{ }^{13} \mathrm{C}$ NMR: (CD$\left.\mathrm{Cl}_{3}, 75 \mathrm{MHz}\right) 21.7\left(4^{\prime \prime \prime}-\mathrm{CH}_{3}\right), 60.1\left(\mathrm{~N}-\mathrm{CH}_{2}\right), 68.5(\mathrm{C}-2), 119.1$ (C$\left.3^{\prime}\right), 120.0\left(\mathrm{C}-8^{\prime}\right), 124.7\left(4 \mathrm{a}^{\prime}\right), 125.4\left(5^{\prime}\right), 128.7\left(\mathrm{C}-3^{\prime \prime}, 5^{\prime \prime}\right), 128.9$ (C2"',6"'), 129.7 (C-3"',5"'), 130.0 (C-2",6"), 132.2 (6'), 133.8 (1"'), 134.0 (C-4"), 134.9 (C-7'), 135.0 (C-1"), 145.2 (C-4"'), 154.6 (C8a'), $155.6\left(\mathrm{C}-2^{\prime}\right), 164.9$ (2-CO), 167.1 (C-1), 175.2 (C-4'). Anal. Calcd for $\mathrm{C}_{26} \mathrm{H}_{20} \mathrm{ClNO}_{7} \mathrm{~S}$ (525.96): C, 59.37; H, 3.83; N, $2.66 \%$. Found: C, $59.51 ; \mathrm{H}, 4.00, \mathrm{~N}, 2.58 \%$.

Data of Compound 3d. White solid, mp $138-140{ }^{\circ} \mathrm{C}$. IR (nujol) $v_{\max }: 3311,1727,1669,1635 \mathrm{~cm}^{-1} .{ }^{1} \mathrm{H}$ NMR: $\left(\mathrm{CDCl}_{3}, 300 \mathrm{MHz}\right) \delta$ : $2.33\left(\mathrm{~s}, 3 \mathrm{H}, 4^{\prime \prime \prime}-\mathrm{CH}_{3}\right), 2.54\left(\mathrm{~s}, 3 \mathrm{H}, 7^{\prime}-\mathrm{CH}_{3}\right), 4.57(\mathrm{dd}, J=14.0,6.0$ $\left.\mathrm{Hz}, 1 \mathrm{H}, \mathrm{N}-\mathrm{CH}_{2}\right), 4.86\left(\mathrm{dd}, J=14.0,7.5 \mathrm{~Hz}, 1 \mathrm{H}, \mathrm{N}-\mathrm{CH}_{2}\right), 6.37(\mathrm{~s}$, $1 \mathrm{H}, \mathrm{H}-2), 7.14$ (d, $\left.J=8.1 \mathrm{~Hz}, 2 \mathrm{H}, \mathrm{H}-3^{\prime \prime \prime}, 5^{\prime \prime \prime}\right), 7.43-7.48$ (m, 2H, H$\left.3^{\prime \prime}, 5^{\prime \prime}\right), 7.47$ (s, $\left.1 \mathrm{H}, \mathrm{H}-8^{\prime}\right), 7.61\left(\mathrm{t}, J=7.5 \mathrm{~Hz}, 1 \mathrm{H}, \mathrm{H}-4^{\prime \prime}\right), 7.67$ (d, $J$ $\left.=8.1 \mathrm{~Hz}, 2 \mathrm{H}, \mathrm{H}-2^{\prime \prime \prime}, 6^{\prime \prime \prime}\right), 8.03-8.05\left(\mathrm{~m}, 2 \mathrm{H}, \mathrm{H}-2^{\prime \prime}, 6^{\prime \prime}\right), 8.07(\mathrm{~s}, 1 \mathrm{H}$, H-5'), 8.14 (s, 1H, H-2'), 9.1 (br m, 1H, NH). ${ }^{13} \mathrm{C}$ NMR: $\left(\mathrm{CDCl}_{3}\right.$, $75 \mathrm{MHz}) 20.1\left(7^{\prime}-\mathrm{CH}_{3}\right), 20.8\left(4^{\prime \prime \prime}-\mathrm{CH}_{3}\right), 59.7\left(\mathrm{~N}^{-} \mathrm{CH}_{2}\right), 67.0(\mathrm{C}-2)$, 118.2 (C-3'), $119.5\left(\mathrm{C}-8^{\prime}\right), 122.0\left(4 \mathrm{a}^{\prime}\right), 124.5\left(5^{\prime}\right), 127.8$ (C-2'", $\left.6^{\prime \prime \prime}\right)$, $128.1\left(\mathrm{C}-3^{\prime \prime}, 5^{\prime \prime}\right), 128.9$ (C-3"',5"'), $129.2\left(\mathrm{C}-2^{\prime \prime}, 6^{\prime \prime}\right), 131.6\left(6^{\prime}\right)$, 132.9 (C-4"), 133.3 (1"'), 133.4 (C-1"), 143.0 (C-7'), 144.2 (C-4"'), $153.7\left(\mathrm{C}-8 \mathrm{a}^{\prime}\right), 155.2\left(\mathrm{C}-2^{\prime}\right), 164.3$ (2-CO), 166.9 (C-1), 173.5 (C$\left.4^{\prime}\right)$. MS (LCMS) $\mathrm{m} / \mathrm{z}(\%) 562 / 564\left(100, \mathrm{M}^{+}+23\right)$. Anal. Calcd for $\mathrm{C}_{27} \mathrm{H}_{22} \mathrm{ClNO}_{7} \mathrm{~S}$ (539.98): C, 60.06; H, 4.11; N, 2.59\%. Found: C, $60.20 ; \mathrm{H}, 4.17, \mathrm{~N}, 2.48 \%$.

Data of Compound 3e. White solid, mp $149-151^{\circ} \mathrm{C}$. IR (nujol) $v_{\max }: 3326,1729,1668,1645 \mathrm{~cm}^{-1} .{ }^{1} \mathrm{H}$ NMR: $\left(\mathrm{CDCl}_{3}+\mathrm{DMSO}_{6}\right.$, $300 \mathrm{MHz}) \delta: 2.37$ (s, 3H, 4"'-- $\left.\mathrm{CH}_{3}\right), 4.62(\mathrm{dd}, J=14.2,5.5 \mathrm{~Hz}, 1 \mathrm{H}$, $\left.\mathrm{N}-\mathrm{CH}_{2}\right), 4.89\left(\mathrm{dd}, J=14.2,8.0 \mathrm{~Hz}, 1 \mathrm{H}, \mathrm{N}-\mathrm{CH}_{2}\right), 6.24(\mathrm{~s}, 1 \mathrm{H}, \mathrm{H}-2)$, $7.21\left(\mathrm{~d}, J=8.1 \mathrm{~Hz}, 2 \mathrm{H}, \mathrm{H}-3^{\prime \prime \prime}, 5^{\prime \prime \prime}\right), 7.47-7.53$ (m, 2H, H-3",5"), 7.5 (br m, 1H, NH), 7.61-7.65 (m, 1H, H-4"), $7.68(\mathrm{~d}, J=9.3 \mathrm{~Hz}, 1 \mathrm{H}$, H-8'), 7.73 (d, $\left.J=8.1 \mathrm{~Hz}, 2 \mathrm{H}, \mathrm{H}-2^{\prime \prime \prime}, 6^{\prime \prime \prime}\right), 8.04-8.09$ (m, 2H, H$\left.2^{\prime \prime}, 6^{\prime \prime}\right), 8.23$ (s, 1H, H-2'), 8.57 (dd, $\left.J=9.3,2.8 \mathrm{~Hz}, 1 \mathrm{H}, \mathrm{H}-7^{\prime}\right), 9.10$ $\left(\mathrm{d}, J=2.8 \mathrm{~Hz}, 1 \mathrm{H}, \mathrm{H}-5^{\prime}\right) \cdot{ }^{13} \mathrm{C}$ NMR: $\left(\mathrm{CDCl}_{3}+\mathrm{DMSO}^{\left.-\mathrm{d}_{6}, 75 \mathrm{MHz}\right)}\right.$

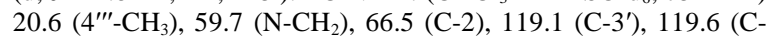
$\left.8^{\prime}\right), 121.3\left(5^{\prime}\right), 122.9\left(4 a^{\prime}\right), 127.6\left(\mathrm{C}-2^{\prime \prime \prime}, 6^{\prime \prime \prime}\right), 127.9\left(\mathrm{C}-3^{\prime \prime}, 5^{\prime \prime}\right), 128.7$ (C-3"',5"'), $129.0\left(\mathrm{C}-2^{\prime \prime}, 6^{\prime \prime}\right), 132.0\left(\mathrm{C}-1^{\prime \prime}\right), 132.8\left(\mathrm{C}-4^{\prime \prime}\right), 132.9$ (C$\left.7^{\prime}\right), 133.4\left(1^{\prime \prime \prime}\right), 145.0\left(\mathrm{C}-4^{\prime \prime \prime}\right), 147.0\left(6^{\prime}\right), 155.8\left(\mathrm{C}-2^{\prime}\right), 158.0$ (C8a'), 164.1 (2-CO), 166.5 (C-1), 173.1 (C-4'). MS (LCMS) m/z (\%) $559\left(100, \mathrm{M}^{+}+23\right)$. Anal. Calcd for $\mathrm{C}_{26} \mathrm{H}_{20} \mathrm{~N}_{2} \mathrm{O}_{9} \mathrm{~S}$ (536.51): $\mathrm{C}$, $58.21 ; \mathrm{H}, 3.76 ; \mathrm{N}, 5.22 \%$. Found: C, 58.07; H,3.67, N, 5.31\%.

Data of Compound 3f. White solid, mp $186-188^{\circ} \mathrm{C}$. IR (nujol) $v_{\max }: 3379,1705,1700,1645 \mathrm{~cm}^{-1} .{ }^{1} \mathrm{H}$ NMR: $\left(\mathrm{CDCl}_{3}+\mathrm{DMSO}_{6}\right.$, $300 \mathrm{MHz}) \delta: 2.33\left(\mathrm{~s}, 3 \mathrm{H}, 4^{\prime \prime \prime}-\mathrm{CH}_{3}\right), 4.55(\mathrm{dd}, J=14.0,5.5 \mathrm{~Hz}, 1 \mathrm{H}$, $\left.\mathrm{N}-\mathrm{CH}_{2}\right), 4.85\left(\mathrm{dd}, J=14.0,7.0 \mathrm{~Hz}, 1 \mathrm{H}, \mathrm{N}-\mathrm{CH}_{2}\right), 6.35(\mathrm{~s}, 1 \mathrm{H}, \mathrm{H}-2)$, 7.12 (d, $\left.J=7.5 \mathrm{~Hz}, 2 \mathrm{H}, \mathrm{H}-3^{\prime \prime \prime}, 5^{\prime \prime \prime}\right), 7.49-7.79$ (m, 6H, H-6', $7^{\prime}, 8^{\prime}$, th$3^{\prime \prime}$,th-4",th-5"), 7.65 (d, $\left.J=8.1 \mathrm{~Hz}, 2 \mathrm{H}, \mathrm{H}-2^{\prime \prime \prime}, 6^{\prime \prime \prime}\right), 8.07$ (s, $1 \mathrm{H}, \mathrm{H}-$ $\left.2^{\prime}\right), 8.20$ (d, $\left.J=7.0 \mathrm{~Hz}, 1 \mathrm{H}, \mathrm{H}-5^{\prime}\right), 9.3$ (br m, $\left.1 \mathrm{H}, \mathrm{NH}\right) .{ }^{13} \mathrm{C}$ NMR: $\left(\mathrm{CDCl}_{3}+\mathrm{DMSO}_{6} \mathrm{~d}_{6}, 75 \mathrm{MHz}\right) 20.4\left(4^{\prime \prime \prime}-\mathrm{CH}_{3}\right), 59.3\left(\mathrm{~N}^{-} \mathrm{CH}_{2}\right), 66.5$ (C-2), $117.3\left(\mathrm{C}-8^{\prime}\right), 117.8\left(\mathrm{C}-3^{\prime}\right), 122.6\left(4 \mathrm{a}^{\prime}\right), 124.6\left(5^{\prime}\right), 124.8\left(6^{\prime}\right)$, 126.9 (th-C-4"), 127.7 (C-2'"',6"'), 128.5 (C-3"',5"'), 131.2 (th-C$\left.2^{\prime \prime}\right), 132.6\left(\mathrm{C}-7^{\prime}\right), 133.1$ (1"'), 133.3 (th-C-3"), 133.5 (th-C-5"), 143.7 (C-4'"'), 154.9 (C-2'), 155.0 (C-8a'), 159.5 (2-CO), 166.5 (C1), $174.0\left(\mathrm{C}^{-} 4^{\prime}\right)$. MS (LCMS) $\mathrm{m} / \mathrm{z}(\%) 520\left(100, \mathrm{M}^{+}+23\right)$. Anal. Calcd for $\mathrm{C}_{24} \mathrm{H}_{19} \mathrm{NO}_{7} \mathrm{~S}_{2}$ (497.54): C, 57.94; H, 3.85; N, $2.82 \%$. Found: C, 58.02; H, 3.77, N, 2.94\%.

Data of Compound 3g. White solid, mp $133-136{ }^{\circ} \mathrm{C}$. IR (nujol) $v_{\max }: 3393,1700,1646 \mathrm{~cm}^{-1} .{ }^{1} \mathrm{H}$ NMR: $\left(\mathrm{CDCl}_{3}, 300 \mathrm{MHz}\right) \delta: 2.29$ (s, 3H, 4'"'- $\mathrm{CH}_{3}$ ), 3.91 (s, 2H, ind-3"- $\mathrm{CH}_{2}$ ), 4.37 (dd, $J=14.1,5.5$ $\left.\mathrm{Hz}, 1 \mathrm{H}, \mathrm{N}-\mathrm{CH}_{2}\right), 4.75\left(\mathrm{dd}, J=14.1,8.2 \mathrm{~Hz}, 1 \mathrm{H}, \mathrm{N}-\mathrm{CH}_{2}\right), 5.96(\mathrm{~s}$, $1 \mathrm{H}, \mathrm{H}-2), 6.99$ (d, $\left.J=7.9 \mathrm{~Hz}, 2 \mathrm{H}, \mathrm{H}-3^{\prime \prime \prime}, 5^{\prime \prime \prime}\right), 7.15$ (ddd, $J=7.7$, $7.2,1.1 \mathrm{~Hz}, 1 \mathrm{H}$, ind-5"), 7.22 (ddd, $J=8.1,7.2,1.2 \mathrm{~Hz}, 1 \mathrm{H}$, ind$\left.6^{\prime \prime}\right), 7.25(\mathrm{~s}, 1 \mathrm{H}$, ind-2"), $7.40(\mathrm{br} \mathrm{d}, J=8.1 \mathrm{~Hz}, 1 \mathrm{H}$, ind-7"), 7.477.53 (m, 2H, H-6', $\left.8^{\prime}\right), 7.52$ (d, $\left.J=7.9 \mathrm{~Hz}, 2 \mathrm{H}, \mathrm{H}-2^{\prime \prime \prime}, 6^{\prime \prime \prime}\right), 7.63$ (br $\mathrm{d}, J=7.7 \mathrm{~Hz}, 1 \mathrm{H}$, ind $\left.-4^{\prime \prime}\right), 7.86\left(\mathrm{~s}, 1 \mathrm{H}, \mathrm{H}-2^{\prime}\right), 7.76$ (ddd, $J=8.5$, 7.2, $\left.1.7 \mathrm{~Hz}, 1 \mathrm{H}, \mathrm{H}-7^{\prime}\right), 8.24$ (dd, $\left.J=8.2,1.7 \mathrm{~Hz}, 1 \mathrm{H}, \mathrm{H}-5^{\prime}\right), 8.38$ (br s, $1 \mathrm{H}, \mathrm{NH}) \cdot{ }^{13} \mathrm{C}$ NMR: $\left(\mathrm{CDCl}_{3}, 75 \mathrm{MHz}\right) 21.6\left(4^{\prime \prime \prime}-\mathrm{CH}_{3}\right), 31.2$ (ind3"- $\left.\mathrm{CH}_{2}\right), 60.0\left(\mathrm{~N}-\mathrm{CH}_{2}\right), 68.3$ (C-2), 107.3 (ind-C-3"), 111.6 (ind-C$\left.7^{\prime \prime}\right), 118.2\left(\mathrm{C}-8^{\prime}\right), 118.7$ (ind-C-4"), 118.7 (C-3'), 119.8 (ind-C-6"), 122.4 (ind-C-5"), 123.6 (ind-C-2"), $123.7\left(4 \mathrm{a}^{\prime}\right), 125.9\left(6^{\prime}\right), 126.0$ (5'), 126.9 (ind-C-3a"), 128.8 (C-2'"',6"'), 129.6 (C-3"',5"'), 133.7 (1"'), 134.5 (C-7'), 136.2 (ind-C-7a"), 145.0 (C-4'"'), 155.8 (C-2'), 156.2 (C-8a'), 167.3 (C-1), 170.2 (2-CO), 176.1 (C-4').

Data of Compound $3 \mathrm{~h}$. White solid, mp $156-158{ }^{\circ} \mathrm{C}$. IR (nujol) $v_{\max }: 3309,1745,1718,1635 \mathrm{~cm}^{-1} .{ }^{1} \mathrm{H}$ NMR: $\left(\mathrm{CDCl}_{3}, 300 \mathrm{MHz}\right) \delta$ : $1.16\left(\mathrm{t}, J=7.5 \mathrm{~Hz}, 3 \mathrm{H}, \mathrm{CH}_{3}\right), 2.33\left(\mathrm{~s}, 3 \mathrm{H}, 4^{\prime \prime \prime}-\mathrm{CH}_{3}\right), 2.42(\mathrm{dq}, J=$ $\left.15.5,7.5 \mathrm{~Hz}, 1 \mathrm{H}, \mathrm{CH}_{2}\right), 2.51\left(\mathrm{dq}, J=15.5,7.5 \mathrm{~Hz}, 1 \mathrm{H}, \mathrm{CH}_{2}\right), 4.46$ $\left(\mathrm{dd}, J=14.1,5.1 \mathrm{~Hz}, 1 \mathrm{H}, \mathrm{N}-\mathrm{CH}_{2}\right), 5.00(\mathrm{dd}, J=14.1,8.4 \mathrm{~Hz}, 1 \mathrm{H}$, N-CH $\left.\mathrm{CH}_{2}\right), 6.06(\mathrm{~s}, 1 \mathrm{H}, \mathrm{H}-2), 7.02\left(\mathrm{~d}, J=8.0 \mathrm{~Hz}, 2 \mathrm{H}, \mathrm{H}-3^{\prime \prime \prime}, 5^{\prime \prime \prime}\right)$, $7.50-7.56\left(\mathrm{~m}, 2 \mathrm{H}, \mathrm{H}-6^{\prime}, 8^{\prime}\right), 7.60\left(\mathrm{~d}, J=8.0 \mathrm{~Hz}, 2 \mathrm{H}, \mathrm{H}-2^{\prime \prime \prime}, 6^{\prime \prime \prime}\right)$, 7.76-7.80 (m, 2H, H-7', NH), 8.01 (s, 1H, H-2'), 8.28 (d, $J=7.8$ $\left.\mathrm{Hz}, 1 \mathrm{H}, \mathrm{H}-5^{\prime}\right) .{ }^{13} \mathrm{C}$ NMR: $\left(\mathrm{CDCl}_{3}, 75 \mathrm{MHz}\right) 8.8\left(-\mathrm{CH}_{2}-\mathrm{CH}_{3}\right), 21.6$ $\left(4^{\prime \prime \prime}-\mathrm{CH}_{3}\right), 27.2\left(-\mathrm{CH}_{2}-\mathrm{CH}_{3}\right), 59.6\left(\mathrm{~N}-\mathrm{CH}_{2}\right), 67.5(\mathrm{C}-2), 118.2(\mathrm{C}-$ $\left.8^{\prime}\right), 118.7\left(\mathrm{C}-3^{\prime \prime \prime}\right), 123.6\left(4 \mathrm{a}^{\prime}\right), 126.0\left(5^{\prime}\right), 126.1\left(6^{\prime}\right), 128.8$ (C$\left.2^{\prime \prime \prime}, 6^{\prime \prime \prime}\right), 129.6\left(\mathrm{C}-3^{\prime \prime \prime}, 5^{\prime \prime \prime}\right), 133.7\left(1^{\prime \prime \prime}\right), 134.6\left(\mathrm{C}-7^{\prime}\right), 145.0$ (C-4"'), 155.2 (C-2'), 156.2 (C-8a'), 167.4 (C-1), 172.8 (2-CO), 176.3 (C$\left.4^{\prime}\right)$. MS (LCMS) $\mathrm{m} / \mathrm{z}(\%) 466\left(100, \mathrm{M}^{++}+23\right)$. Anal. Calcd for $\mathrm{C}_{22} \mathrm{H}_{21} \mathrm{NO}_{7} \mathrm{~S}$ (443.47): C, 59.58; H, 4.77; N, 3.16\%. Found: C, $59.70 ; \mathrm{H}, 4.87, \mathrm{~N}, 3.05 \%$.

[20] Synthesis of 2-ethoxy- $N$-(ethoxymethyl)-2-(4-oxo-4H-chromen3-yl)acetamide 4a. A solution of $\mathrm{NaOH}(50 \mathrm{mg})$ in $\mathrm{EtOH},(5 \mathrm{~mL})$ was added to a solution of $3 \mathrm{a}(0.5 \mathrm{mmol})$ in $\mathrm{EtOH}(1 \mathrm{~mL})$. The mixture was stirred at room temperature for $24 \mathrm{~h}$, the volume was reduced to $1 \mathrm{~mL}$, whereupon the solid, which precipitated, was filtered and washed initially with water and then with cold ethanol, yielding compound $\mathbf{4 a}$ in an essentially pure form. Yield, $71 \%$, white solid; IR (nujol) $v_{\max }: 1623 \mathrm{~cm}^{-1} .{ }^{1} \mathrm{H}$ NMR: $\left(\mathrm{CDCl}_{3}, 300\right.$ MHz) $\delta: 1.18\left(\mathrm{t}, J=7.0 \mathrm{~Hz}, 3 \mathrm{H}, \mathrm{CH}_{3}\right), 1.30(\mathrm{t}, J=7.0 \mathrm{~Hz}, 3 \mathrm{H}$, $\left.\mathrm{CH}_{3}\right), 3.56\left(\mathrm{dq}, J=7.0,4.5 \mathrm{~Hz}, 1 \mathrm{H}, \mathrm{CH}_{2}\right), 3.58(\mathrm{dq}, J=7.0,4.5 \mathrm{~Hz}$, $\left.1 \mathrm{H}, \mathrm{CH}_{2}\right), 3.63\left(\mathrm{dq}, J=7.0,4.5 \mathrm{~Hz}, 1 \mathrm{H}, \mathrm{CH}_{2}\right), 3.65(\mathrm{dq}, J=7.0,4.5$ $\left.\mathrm{Hz}, 1 \mathrm{H}, \mathrm{CH}_{2}\right), 4.716\left(\mathrm{dd}, J=10.3,7.0 \mathrm{~Hz}, 1 \mathrm{H}, \mathrm{N}-\mathrm{CH}_{2}\right), 4.816(\mathrm{dd}$, $\left.J=10.3,7.0 \mathrm{~Hz}, 1 \mathrm{H}, \mathrm{N}-\mathrm{CH}_{2}\right), 4.92(\mathrm{~d}, J=0.75 \mathrm{~Hz}, 1 \mathrm{H}, \mathrm{H}-2), 7.43$ (ddd, $\left.J=8.0,7.0,1.0 \mathrm{~Hz}, 1 \mathrm{H}, \mathrm{H}-6^{\prime}\right), 7.47(\mathrm{dd}, J=8.5,1.0 \mathrm{~Hz}, 1 \mathrm{H}$, H-8'), 7.69 (ddd, $\left.J=8.5,7.0,1.75 \mathrm{~Hz}, 1 \mathrm{H}, \mathrm{H}-7^{\prime}\right), 7.75$ (br m, 1H, $\mathrm{NH}), 8.04\left(\mathrm{~d}, J=0.75 \mathrm{~Hz}, 1 \mathrm{H}, \mathrm{H}-2^{\prime}\right), 8.21(\mathrm{dd}, J=8.0,1.75 \mathrm{~Hz}$, 1H, H-5'). ${ }^{13} \mathrm{C}$ NMR: $15.1\left(\mathrm{CH}_{3}\right), 15.2\left(\mathrm{CH}_{3}\right), 64.1\left(\mathrm{CH}_{2}\right), 66.0$ $\left(\mathrm{CH}_{2}\right), 69.7(\mathrm{C}-2), 74.7\left(\mathrm{~N}^{-} \mathrm{CH}_{2}\right), 118.2\left(\mathrm{C}-8^{\prime}\right), 121.3\left(\mathrm{C}-3^{\prime}\right), 124.0$ (C-4a'), 125.5 (C-5'), 126.0 (C-6'), $134.0\left(\mathrm{C}^{\prime} 7^{\prime}\right), 155.0\left(\mathrm{C}-2^{\prime}\right), 156.3$ (C-8a'), 170.8 (C-1), 176.5 (C-4'). MS (LCMS) m/z (\%) 328 (100, $\mathrm{M}^{++}+23$ ). Anal. Calcd for $\mathrm{C}_{16} \mathrm{H}_{19} \mathrm{NO}_{5}$ (305.33): C, 62.94; H, 6.27; $\mathrm{N}, 4.59 \%$. Found: C, 63.16; H, 6.40, N, $4.38 \%$.

Data of Compound $4 \mathrm{c}$. Yield, $74 \%$, white solid, mp $177-179^{\circ} \mathrm{C}$ $\left(\mathrm{CH}_{2} \mathrm{Cl}_{2}\right.$-pet. ether). ${ }^{1} \mathrm{H} \mathrm{NMR}$ : $\left(\mathrm{CDCl}_{3}, 300 \mathrm{MHz}\right) \delta: 1.20$ (t, $J=7.0$ $\left.\mathrm{Hz}, 3 \mathrm{H}, \mathrm{CH}_{3}\right), 1.27\left(\mathrm{t}, J=7.0 \mathrm{~Hz}, 3 \mathrm{H}, \mathrm{CH}_{3}\right), 3.55(\mathrm{dq}, J=7.0,4.5$ $\left.\mathrm{Hz}, 1 \mathrm{H}, \mathrm{CH}_{2}\right), 3.57\left(\mathrm{dq}, J=7.0,4.5 \mathrm{~Hz}, 1 \mathrm{H}, \mathrm{CH}_{2}\right), 3.63(\mathrm{dq}, J=$ $\left.7.0,4.5 \mathrm{~Hz}, 1 \mathrm{H}, \mathrm{CH}_{2}\right), 3.65\left(\mathrm{dq}, J=7.0,4.5 \mathrm{~Hz}, 1 \mathrm{H}, \mathrm{CH}_{2}\right), 4.80(\mathrm{~d}$, $\left.J=6.8 \mathrm{~Hz}, 2 \mathrm{H}, \mathrm{N}_{-} \mathrm{CH}_{2}\right), 4.95(\mathrm{~s}, 1 \mathrm{H}, \mathrm{H}-2), 7.43(\mathrm{~d}, J=9.0 \mathrm{~Hz}, 1 \mathrm{H}$, H-8'), 7.62 (dd, $\left.J=9.0,2.5 \mathrm{~Hz}, 1 \mathrm{H}, \mathrm{H}-7^{\prime}\right), 7.70$ (br m, 1H, NH), $8.02\left(\mathrm{~s}, 1 \mathrm{H}, \mathrm{H}-2^{\prime}\right), 8.16\left(\mathrm{~d}, J=2.5 \mathrm{~Hz}, 1 \mathrm{H}, \mathrm{H}-5^{\prime}\right) .{ }^{13} \mathrm{C}$ NMR: 15.1 $\left(\mathrm{CH}_{3}\right), 15.2\left(\mathrm{CH}_{3}\right), 64.1\left(\mathrm{CH}_{2}\right), 66.2\left(\mathrm{CH}_{2}\right), 69.7(\mathrm{C}-2), 74.6(\mathrm{~N}-$ $\left.\mathrm{CH}_{2}\right), 119.9\left(\mathrm{C}-8^{\prime}\right), 121.4\left(\mathrm{C}-3^{\prime}\right), 124.9\left(\mathrm{C}-4 \mathrm{a}^{\prime}\right), 125.4\left(\mathrm{C}-5^{\prime}\right), 131.5$ (C-6'), 134.3 (C-7'), 154.7 (C-8a'), 155.1 (C-2'), 170.6 (C-1), 175.4 $\left(\mathrm{C}-4^{\prime}\right)$. MS (LCMS) m/z (\%) $328\left(100, \mathrm{M}^{+}+23\right)$. Anal. Calcd for $\mathrm{C}_{16} \mathrm{H}_{18} \mathrm{ClNO}_{5}$ (339.77): C, 56.56; H, 5.34; N, 4.12\%. Found: $\mathrm{C}$, $56.39 ; \mathrm{H}, 5.42, \mathrm{~N}, 4.18 \%$. 